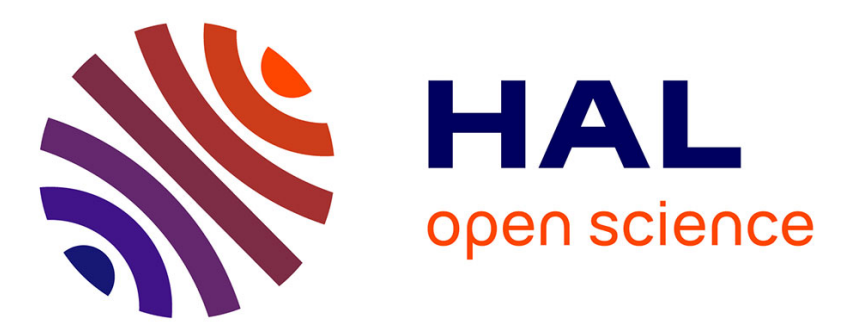

\title{
Numerical modeling of bone as a multiscale poroelastic material by the homogenization technique
}

Eléonore Perrin, Benyebka Bou-Saïd, Francesco Massi

\section{To cite this version:}

Eléonore Perrin, Benyebka Bou-Saïd, Francesco Massi. Numerical modeling of bone as a multiscale poroelastic material by the homogenization technique. Journal of the mechanical behavior of biomedical materials, 2019, 91, pp.373 - 382. 10.1016/j.jmbbm.2018.12.015 . hal-03487039

\section{HAL Id: hal-03487039 \\ https://hal.science/hal-03487039}

Submitted on 20 Dec 2021

HAL is a multi-disciplinary open access archive for the deposit and dissemination of scientific research documents, whether they are published or not. The documents may come from teaching and research institutions in France or abroad, or from public or private research centers.
L'archive ouverte pluridisciplinaire $\mathbf{H A L}$, est destinée au dépôt et à la diffusion de documents scientifiques de niveau recherche, publiés ou non, émanant des établissements d'enseignement et de recherche français ou étrangers, des laboratoires publics ou privés.

\section{다)(1) $(5$}

Distributed under a Creative Commons Attribution - NonCommerciall 4.0 International 


\title{
Numerical modeling of bone as a multiscale poroelastic material by the homogenization technique
}

\author{
Eléonore Perrin (1) (2), Benyebka Bou-Saïd (1), Francesco Massi (2)
}

1. LaMCoS, INSA Lyon, CNRS, UMR5259, University of Lyon, Villeurbanne, France; 2. DIMA, University of Rome, La Sapienza, Rome, Italy

\begin{abstract}
Bone is a complex material showing a hierarchical and porous structure but also a natural ability to remodel thanks to cells sensitive to fluid flows. Based on these characteristics, a multiscale numerical model has been developed in order to represent the bone response under mechanical solicitation. It relies on the homogenization technique, simulating bone as a homogeneous structure having a porous microstructure saturated with bone fluid. The numerical modeling of the loading of a finite volume of bone enables the determination of an equivalent poroelastic stiffness. Focusing on two extreme fluid boundary conditions, the study of the corresponding structural response provides an overview of the fluid contribution to the poroelastic behavior, impacting the stiffness of the considered material. This parameter is either reduced (when the fluid can flow out of the structure) or increased (when the fluid is kept inside the structure) and quantified through this model. The presented poroelastic numerical model is here developed in the perspective of providing a bio-reliable model of bones, to determine the critical parameters that might impact bone remodeling.
\end{abstract}

\section{Keywords}

Bone model, poroelasticity, multiscale model, homogenization technique, mechanotransduction 


\section{Nomenclature}

$b$ : Biot coefficient

$C$ : Elastic stiffness tensor

$C_{e f f}$ : Effective elastic stiffness tensor

$C_{f}:$ Forchheimer coefficient properties

$D\left(\overrightarrow{v_{f}}\right)$ : Strain rate tensor in the fluid part

$E$ : Young's Modulus

$E\left(\overrightarrow{\left.u_{s}\right)}\right.$ : Strain tensor in the solid part

$K_{s}$ : Bulk modulus of the solid part

$K_{b}$ : Bulk modulus of the drained skeleton

$K_{f}$ : Bulk modulus of the fluid part

$k$ : Permeability coefficient

$Q$ : Biot modulus

$q$ : Derivative of the expression for the fluid accumulation

$p_{f}$ : pressure of the fluid

$\overrightarrow{u_{s}}$ : displacement in the solid part

$\overrightarrow{v_{f}}$ : velocity of the fluid

$\alpha$ : Biot effective stress coefficient tensor

$\delta$ : Kronecker symbol

$\varepsilon:$ scale ratio between the microscopic and the macroscopic scale

$\vec{\eta}$ : Microscopic solid displacement vector within the elementary cell due to a unit value of the liquid pressure

$\mu$ : Fluid viscosity.

$\mu_{\text {eff }}$ : Effective viscosity parameter

$v$ : Poisson's coefficient

$\vec{\xi}$ : Microscopic solid displacement vector within the elementary cell due to a unit value of the macroscopic strain tensor

$\rho_{f / s}$ : Density of the fluid or the solid respectively

$\sigma_{s / f}:$ respectively stress tensor in the solid and in the fluid

$\Omega_{\mathrm{S} / \mathrm{f}}$ : respectively the solid part and the fluid part

$\phi$ : porosity of the poroelastic material

$\nabla$ : gradient operator

$\nabla_{\mathrm{x}}$ : gradient operator for the macroscopic (also referred as global) scale 
$\nabla_{\mathrm{y}}$ : gradient operator for the microscopic (also referred as local) scale 


\section{INTRODUCTION}

Bone is known to be a particularly complex material. It can be a multiscale material as it possesses a hierarchical structure [1] [2] [3]. From the nanoscale to the macroscale, each intermediate scale is composed of a specific pattern. The pattern consists of a periodic elementary structure of the larger scale. Down to the nanoscale, bone is a composite material, with a mineral phase embedded in an organic matrix.

Furthermore, as bone is a vascularized tissue, each scale presents an individual porosity, differing in pore shape and porosity values. Depending on the scale considered, the pores are filled with bone marrow, blood, or interstitial fluid and cells.

All these particularities represent many potential parameters in the development of a numerical model of bone and have been the subject of many experimental investigations. The latter are usually done by nanoindentation measures, and bone elastic modulus at this scale can vary between 5 and 25GPa [1] [4] [5] [6] [7] or by micro-CT analysis [8] allowing bone quality characterization. This large range of value can be explained by the non-linear stress-strain behavior of the collagen fibrils contained in bone internal structure as highlighted in [9]. The presence of fluid in bone also impact its mechanical behavior but is very difficult to characterize experimentally. However, several attempts have been made, through water imbibition of bone samples [10] or internal medullary pressure measurements as in [11] among others.

Thus, transposing bone actual mechanical specificities in numerical models is a real challenge. Indeed, bone material can be seen either as a composite material [3], or as a viscoelastic material due to the viscous aspect of bone marrow [12], or as a poroelastic material taking into account the different levels of porosity and the influence of the corresponding fluid flow [13].

Keeping in mind all these considerations, one needs to have a precise idea of the final aim and application of the bone numerical modeling in its development, to choose the most relevant working hypotheses. In this work, the focus is on the natural ability of bone to adapt its structure according to its mechanical and biological environment. This is called bone remodeling, and it is enabled by the mechanosensitive bone cells that orchestrate the surrounding cellular activity resulting in bone adaptation. Such bone cells, called osteocytes, are immersed in the interstitial fluid and located in the bone porosity found at the microscale, in the lacunar-canalicular system [14] [15].

Bone remodeling is particularly involved in bone healing after implantation of an articular prothesis during, for example, a Total Hip Arthroplasty. A successful implantation is quantified by the strength of the bond created between the prosthesis and the host bone without causing thigh pain or prothesis loosening for the patient. Many parameters are involved in pre-, per- and post-surgery: prosthesis shape, position in the femur, post-surgery loading among many others. To determine which parameters are particularly relevant in bone-implant long-term stability, the investigation of bone mechanical behavior and bone remodeling in this context is a major socio-economic enterprise.

In this specific context, an appropriate choice in the focused bone characteristics is required to maintain a relevant compromise between precision and computational time. As internal fluid flows are essential in the structural constitution of bone and in osteocyte activation, a focus is placed on bone poroelastic aspects, including the fluid presence within the pores. The aim is to provide a better understanding of the events occurring at the microscale, and the impact of those local events on the structural and remodeling behavior.

Therefore, considering bone as a poroelastic material gives interesting outlines. Poroelasticity refers to a theoretical model of the mechanical behavior of a porous structure filled with fluid. It first has been conceptualized and formulated by Biot in the 40's and its work on soil consolidation [16] [17]. From a numerical point of view, several approaches can be considered, such as effective medium theory [18], mixture theory [19] [20] or a multiscale approach. Through homogenization theory, developed since 1978 [21], one is able consider both periodic and random sub-structures in a heterogeneous material mechanical behavior [22]. The corresponding scope of application is very large [23], including 
composite material modeling, but also investigation of porous materials, following both linear and nonlinear poroelasticity laws [24].

Keeping in mind the final aim of the developed model presented here, multiscale modeling appears to the authors as the most adapted method, allowing a precise consideration of local events with reasonable computational costs.

The microstructure modeled here, being the lacunar-canalicular system, can be assimilated to a periodic structure and the application of a homogenization technique is then performed [25]. This is a numerical procedure elaborated in [21] and largely developed since, based on the assumption of scale separation. This approach has been already proven relevant in the context of bone modeling [26] [27].

Indeed, several works can be found in the literature modeling bone as a poroelastic material with a multiscale formulation, but the different models are not developed for the same purpose. Some works [26] [11] bring a better understanding of osteocytes activation, while in [27] the model is dedicated to predict the qualitative changes in anisotropy due to variations in the structure at the mesoscale. In [29], [29], [30] and [31] this formulation of modeling is used to produce a complete and rigorous model of bone aimed to a solid bio-reliability, considering several porosities and not only the hydraulic component of osteocytes activation. The multiple porosity aspects and anisotropic properties of bone can be introduced as well, e.g. in [32] [33] and [34], to thoroughly enlarge the scope of application.

Nevertheless, its application in the elaboration of a numerical modeling based on a compromise between accuracy and efficiency has not yet been considered to our knowledge. The aim is here to provide a numerical tool, easy to put in place and computationally efficient, for bone reconstruction prediction for both the physician and the prosthesis manufacturer. The main issue is therefore to reach an equilibrium between efficiency and accuracy at the numerical level. This result in the formulation of a multiscale poroelastic model of bone is searched through the homogenization technique for periodic structures, considering the device scale (referred as the macroscale) and the level of the events involved in bone reconstruction, i.e. the cellular level at the macroscale. First the governing equations and the modelling strategy are described. Then, results from the simulations obtained by the developed model are presented and discussed. 


\section{MULTISCALE POROELASTIC MODEL DEVELOPMENT}

As in any biological structure, a perfect periodicity cannot be achieved regarding the continuous remodeling of bone tissue. However, in the context of cortical bone in the femur, where the structure is notably organized along the principal mechanical loading, the regularity of the canaliculi and the lacunae is a reasonable assumption [35] [36] for the scope of the proposed modelling.

\subsection{Model DeVelopment}

\subsubsection{Representative Volume Element definition}

In order to settle the framework of the homogenization technique in the context of poroelastic modeling, an appropriate RVE needs to be carefully chosen.

The RVE must represent the microstructure of the poroelastic material under consideration. Keeping in mind the final aim of the developed model, and considering the structure of the bone matrix, the pore shape is composed of communicating channels. The size is determined from the biological dimension of the lacunar-canalicular system that has to be modeled, corresponding to a porosity $\phi=5 \%$ [13].

It is composed of a solid and a fluid part. The solid part (referred as $\Omega_{\mathrm{s}}$ ) is considered elastic, isotropic and evolving according to the small deformation assumption. Regarding its geometry, at this scale, the porosity of the bone is estimated at $5 \%$ and the diameter of the considered canals considered is on the order of $0.3 \mu \mathrm{m}$ [13]. Thus, the poroelastic RVE is a drilled cube of $100 \mu \mathrm{m}$ length with $10 \mu \mathrm{m}$ channels, as shown in Figure 1. The fluid part (referred as $\Omega_{\mathrm{f}}$ ) represents bone fluid and saturates the pores. As it is mainly composed of water, it is considered incompressible and Newtonian. Its viscosity is estimated to the one of saline water at $37^{\circ} \mathrm{C}$.

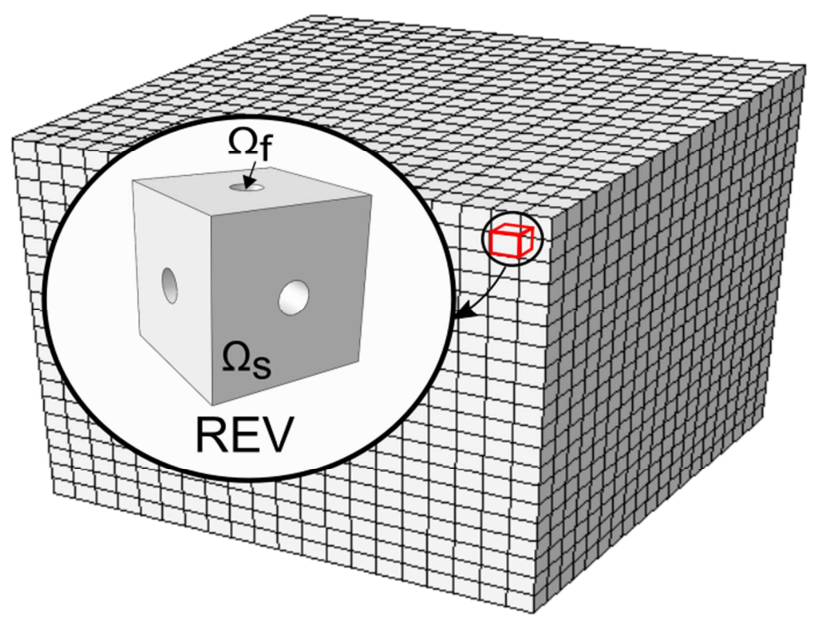

Figure 1 - Poroelastic REV composed of a solid domain $\Omega_{\mathrm{s}}$ and a fluid domain $\Omega_{\mathrm{f}}$.

\subsubsection{Mathematical development}

\subsubsection{Conservation laws}

In $\Omega_{\mathrm{s}}$, without external forces and under the assumption of small deformations, the equation of motion is written:

$$
\nabla \cdot \sigma_{s}=\overrightarrow{0}
$$


where $\sigma_{s}$ is the stress tensor, defined as $\sigma_{s}=C: E\left(\overrightarrow{u_{s}}\right)$ with $C$ the elastic tensor of the solid part, $E\left(\overrightarrow{u_{s}}\right)$ the strain tensor and $\overrightarrow{u_{s}}$ the displacement vector [37].

In $\Omega_{\mathrm{f}}$, without external forces, the equations of motion are written:

$$
\nabla . \sigma_{f}=\overrightarrow{0}
$$

where $\sigma_{f}$ is the stress tensor, defined as $\sigma_{f}=-p_{f} \delta+2 \mu D\left(\overrightarrow{v_{f}}\right)$.

In this expression, $p_{f}$ is the pressure if the fluid, $\mu$ its viscosity, $\delta$ is the identity tensor, $D\left(\overrightarrow{v_{f}}\right)$ the strain rate tensor and $\overrightarrow{v_{f}}$ the velocity vector of the fluid. This latter is defined by the following equation of motion:

$$
\nabla \cdot \overrightarrow{v_{f}}=q
$$

where $q$ represents the derivative of the expression for the fluid accumulation in the RVE defined by:

$$
q=\frac{1}{Q} \dot{p_{f}}+b \operatorname{Tr}\left(\dot{E\left(\overrightarrow{\left.u_{s}\right)}\right)}\right.
$$

Equation (4) involves $Q$, the Biot modulus, that considers the compressibility and $b$, the Biot coefficient, expressed with the bulk modulus of the drained solid matrix and the solid matrix itself [38]:

$$
\begin{gathered}
Q=\left(\frac{b-\phi}{K_{b}}+\frac{\phi}{K_{f}}\right)^{-1} \\
b=1-\frac{K_{b}}{K_{s}}
\end{gathered}
$$

where $K_{s}, K_{b}$ and $K_{f}$ are, respectively, the bulk modulus of the solid part, of the drained skeleton and of the fluid phase.

At the interface, called $\Gamma$, continuity conditions are applied, with $\vec{n}$ is the normal vector to the interface:

$$
\begin{aligned}
\overrightarrow{v_{f}} & =\frac{\partial \overrightarrow{u_{s}}}{\partial t} \\
\sigma_{f} \vec{n} & =\sigma_{s} \vec{n}
\end{aligned}
$$

\subsubsection{Application of the homogenization technique}

From the conservation laws previously presented, the three unknown variables of the system are $\overrightarrow{u_{s}}$, $p_{f}$ and $\overrightarrow{v_{f}}$. According to the homogenization technique previously described, these three variables are written in the form of an asymptotic expansion of the first order in powers of $\varepsilon$, precise enough for the mathematical set up of homogenized conservation laws, $\varepsilon$ being the scale ratio $\left(\varepsilon=\frac{l}{L}<<1, l\right.$ being the representative length of the microscale, $L$ the one of the macroscales). It gives:

$$
\begin{aligned}
& \overrightarrow{u_{s}}={\overrightarrow{u_{s}}}^{0}(x, y, t)+\varepsilon{\overrightarrow{u_{s}}}^{1}(x, y, t)+o\left(\varepsilon^{2}\right) \\
& \overrightarrow{v_{f}}={\overrightarrow{v_{f}}}^{0}(x, y, t)+\varepsilon{\overrightarrow{v_{f}}}^{1}(x, y, t)+o\left(\varepsilon^{2}\right) \\
& p_{f}=p_{f}{ }^{0}(x, y, t)+\varepsilon p_{f}^{1}(x, y, t)+o\left(\varepsilon^{2}\right)
\end{aligned}
$$

The exponents are referring to the considered orders. The homogenization technique also involves the use of the expression of the gradient operator [21] [39], where the subscripts $x$ and $y$ are respectively referring at the macroscale and the microscale: 


$$
\nabla=\nabla_{x}+\frac{1}{\varepsilon} \nabla_{y}
$$

\subsubsection{Final equations}

Incorporating the asymptotic expansions (9), (10), (11) into the expressions (1), (2), (3) using the expression (12), averaging over the elementary cell ( $<*>=\frac{1}{\Omega} \int * d \Omega$ where $\Omega=\Omega_{s} \cup \Omega_{f}$ ), the macroscopic equations resulting from the homogenization theory allow the expression of the stress in the macroscopic poroelastic material $\left(<\sigma_{\text {poro }}>\right)$ and are written:

$\ln \Omega_{\mathrm{s}}$ :

$$
\begin{gathered}
\nabla_{x}<\sigma_{\text {poro }}>=\overrightarrow{0} \\
<\sigma_{\text {poro }}>=C^{e f f} E_{x}\left(\overrightarrow{u_{s}^{0}}\right)-\alpha p_{f}^{0}
\end{gathered}
$$

$C^{e f f}$ and $\alpha$ are the effective elastic stiffness tensor and the Biot effective stress coefficient resulting from the local RVE problem and $E_{x}\left(\overrightarrow{u_{s}^{0}}\right)$ is the strain tensor at the macroscale. They are expressed as in the following:

$$
\begin{aligned}
C_{i j k l}^{e f f} & =<C_{i j k l}+C_{i j m n}: E_{y m n}(\overrightarrow{\xi k l})> \\
\alpha_{i j} & \left.=\phi \delta_{i j}+<C_{i j m n}: E_{y m n} \overrightarrow{(\eta)}\right)>
\end{aligned}
$$

In the expressions (15) and (16), $\vec{\xi}$ and $\vec{\eta}$ are two particular solutions of the equilibrium Equation (17). It is reminded that we are looking for $C^{e f f}$ and $\alpha_{i j}$, thus we solve the system applying two sets of particular boundary conditions (18) and (19) and $E_{y}(\vec{\xi})$ and $E_{y}(\vec{\eta})$ are the corresponding microscopic strain tensor:

$$
\begin{gathered}
\nabla_{y} \cdot\left[C: E_{y}\left(\overrightarrow{u_{s}^{1}}\right)+C: E_{x}\left(\overrightarrow{u_{s}^{0}}\right)\right]=0 \\
\left(p_{f}^{0}=0 ; E_{x}\left(\overrightarrow{u_{s}^{0}}\right)=\frac{1}{2}\left(\delta_{j l} \cdot \delta_{k m}+\delta_{j m} \cdot \delta_{k l}\right)\right. \\
\left(p_{f}^{0}=1 ; E_{x}\left(\overrightarrow{u_{s}^{0}}\right)=0\right)
\end{gathered}
$$

$\ln \Omega_{\mathrm{f}}:$

$$
\begin{gathered}
\nabla_{x} \cdot \overrightarrow{v_{f}^{0}}>=0 \\
<\overrightarrow{v_{f}^{0}}>=-\frac{k}{\mu} \nabla_{x} p_{f}^{0}
\end{gathered}
$$

Equation (21) is the Darcy's law that can be completed by other terms in order to fit at best with fluid conditions [40]. It can include the Forchheimer's term, representing the inertial effects

$$
\left.\nabla_{x} p_{f}^{0}=-\frac{\mu}{k} \overrightarrow{v_{f}^{0}}-\frac{\rho C_{f}}{\sqrt{k}} \overrightarrow{\left(v_{f}^{0}\right.}\right)^{2}
$$

It requires the determination of a parameter called the Forchheimer coefficient $C_{f}$, believed to be fixed for a given class of porous media and dimensions [41] and the fluid density $\rho$.It can also include the Brinkman's term (Expression (23)) that considers interface phenomena between the fluid and the wall of the pores [42]. 


$$
\nabla_{\mathrm{x}} p_{f}^{0}=-\frac{\mu}{k} \overrightarrow{v_{f}^{0}}+\mu_{e f f} \overrightarrow{\Delta v_{f}^{0}}
$$

$\Delta$ is the Laplacian operator. Therefore, as it involves the second derivative of the velocity, the relevancy of the Darcy-Brinkman model is determined by the velocity variations within the fluid flow. It can be noted that although the determination of the effective viscosity and its consistency is widely discussed in the literature, one consensus is reached regarding its relation to porosity, namely, that $\mu_{e f f}$ is only relevant when considering materials characterized by high porosity [43] [44].

The relevancy of both terms needs to be discussed regarding the context of application. 


\subsection{Algorithm}

In order to transpose these equations to a numerical model, an algorithm is put in place. It is articulated through the commercial softwares Abaqus and MATLAB.

\section{INPUTS}

In the solid part

1. Material properties of the solid matrix

1. Boundary conditions at the macroscopic scale

In the fluid part

- Material properties of the fluid i

2. RESOLUTION OF THE RVE PROBLEM

Determination of the effective properties $C_{\text {eff }}$ and $\alpha$
Finite-Element Method
Abaqus

3. COMPUTATION OF THE PRESSURE

Link by Python Scripts

Determination of $p_{f}^{0}$

- Computation of the pressure, accounting for the fluid accumulation in the cube

4. COMPUTATION OF THE STRESS

Calculation of the stress in the homogenized and poroelastic

material

- Homogenized and macroscopic expression of the stress $\left\langle\sigma_{\text {poro }}\right\rangle$

$$
<\sigma_{\text {poro }}>=C^{e f f} E_{x}\left(\overrightarrow{u_{s}^{0}}\right)-\alpha p_{f}^{0}
$$

Finite-Difference Method MATLAB

Link by Python Scripts

Method

Abaqus (Subroutine)

\section{IF CONVERGENCE NOT REACH $\rightarrow$ Back in step 3}

Convergence is estimated calculated the pressure variation between two iterations.

Once it is reached, pressure is computed without external force (loading or structural strain) to let fluid stabilization occurring.

5. OUTPUTS

In the solid part

- Stress and Strain distributions in the poroelastic material

- Displacements

- Apparent Young Modulus
In the fluid part

- Pressure distribution

Figure 2 - Global solution algorithm. 


\section{APPLICATION TO CORTICAL BONE SIMULATION}

In the following the computation of each step of the algorithm is described, when simulating the compression of a cortical bone sample.

\subsection{STEP 1: INPUTS}

The porosity considered here corresponds to the lacunar-canalicular porosity found at the cellular level. The porosity contains the mechanosensitive cells, responsible for the equilibrium of bone remodeling. In this specific case, the chosen material characteristics are detailed in Table 1. They correspond to characteristics of cortical bone elastic and interstitial fluid found in the literature [13].

\begin{tabular}{|c|c|c|c|}
\hline \multicolumn{2}{|c|}{ Solid part: elastic cortical bone } & \multicolumn{2}{|c|}{ Fluid part: Interstitial fluid } \\
\hline E (Young's Modulus) & $14000 \mathrm{MPa}$ & \multirow{4}{*}{$\mu$ (Dynamic viscosity) } & \multirow{4}{*}{$\begin{array}{c}0.0065 \text { (Pa.s) } \\
\text { (salt water at } 37^{\circ} \mathrm{C} \text { ) }\end{array}$} \\
\hline$\rho_{s}$ (Density of the solid part) & $1.6 \mathrm{~g} / \mathrm{cm}^{3}$ & & \\
\hline$v$ (Poisson's coefficient) & 0.3 & & \\
\hline k (Material Permeability) & $1.5 \times 10^{-14} \mathrm{~mm}^{2}$ & & \\
\hline
\end{tabular}

Table 1 - Cortical bone material properties used as input in the poroelastic model [13].

As a case study, a finite volume of bone, corresponding to a cube of $2 \times 2 \mathrm{~mm}$, is simulated under compression, with a pressure ramp applied on the top face (Figure 3). The cube is considered clamped at its bottom face.

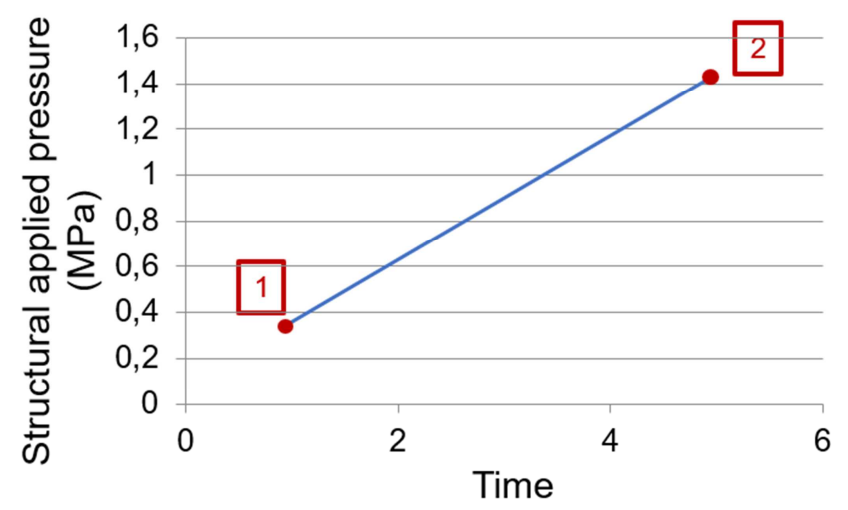

Figure 3 - Pressure ramp applied on the top face. The results are extracted at point 1 , when the loading pressure is 0.36 $\mathrm{MPa}$, and at point 2, when the loading pressure is $1.44 \mathrm{MPa}$.

\subsection{Step 2: Resolution OF THE RVE PROBLEM}

Applying the specific boundary conditions deduced from the homogenization technique (see Equations (18) and (19)) the effective properties $C_{e f f}$ and $\alpha$ are determined for the bone material and implemented in the macroscopic poroelastic model. In order to fit homogenization technique assumptions, periodic boundary conditions are applied on the external faces.

This resolution is made within the Abaqus software, using Abaqus meshing options for tetrahedral elements. The (18) boundary condition is applied through a subroutine imposing a strain field and the (19) boundary condition is applied on $\Gamma$.

\subsection{Step 3: ComputATION OF THE PRESSURE}

To choose an accurate formulation of the evolution of the pressure in the bone, an analysis of a critical criterion is performed to ensure the relevancy of each term. In the context of clinical application of the developed model, and for the design of devices such as a hip prosthesis, a critical loading phase on this joint happens during activities such as climbing stairs or rising from a chair. This critical case can be likened to a $1 \mathrm{~mm}$ length cube under a compression load of $18 \mathrm{MPa}$ [45]. 
This gives the information required to compute the Reynolds number (found in most cases to be less than one) and to state the fact that inertial effects are neglectable in the femur, in a specific mechanically demanding situation. The use of the Forchheimer term in the computation of the pressure is therefore not necessary. Concerning the Brinkman term, as the working porosity is $5 \%$, it can be as well excluded.

Thus, the fluid pressure and the fluid velocity in the cortical bone are calculated with Darcy's law added to the fluid accumulation term, as reported in Equation (21). It is calculated by a finite differences method on Matlab.

\subsection{STEP 4: COMPUTATION OF THE STRESS}

After these three steps, all the elements are gathered to compute the stresses in the homogenized poroelastic material. The calculated quantities are introduced in an UMAT subroutine in Abaqus, coded in Fortran and aiming to compute the stress according to the Expression (14).

\subsection{OUtPuts and Numerical Validation}

Once the multiscale poroelastic model of bone, with the appropriate input data for cortical bone, is fully developed, its consistency regarding its final aim can be tested by comparison with an isotropic and elastic model.

The main advantage being the inclusion of the fluid part, it is now possible to evaluate the impact of this component on the macroscopic response of the material.

The aim here is to propose a first model validation, analyzing the results from a simple case study and checking the numerical consistency of the proposed model, as a preliminary study. A simple geometry of the simulated volume has been chosen to evaluate the consistence of the simulation results, as a function of the boundary conditions, within a volume representative of the investigated scale.

To do so, two extreme and opposite cases for the fluid boundary conditions are here considered: either the fluid is completely trapped in the poroelastic material, either it can completely flow out. The reference pressure is set to zero implying that at the beginning of the simulations, the pressure in all the pores is equal to zero.

The corresponding boundary conditions in fluid can be described as follows:

(i) a case where the cube walls are impermeable, and the fluid cannot flow out;

(ii) a case where the reference pressure is considered at the walls, allowing the fluid to flow out from the structure.

The same structural boundary conditions are applied within the Abaqus interface: an arbitrary linear increase of the loading pressure, from zero to $1.44 \mathrm{MPa}$, is applied on the top face of a $2 \mathrm{~mm}$ length cube, whose bottom face is clamped, modeling a reproducible experimental test for further experimental validation, while external walls are kept free from a structural point of view. The material properties are those presented in Table 1. The corresponding equivalent values of Young's modulus, for the two simulated boundary conditions on the fluid, are estimated from the obtained displacements.

\subsubsection{Pressure evolution and structural deformation}

Under the set of boundary conditions of the case (i), the results presented on Figure 4 are obtained. The pressure distribution, the corresponding structural stress in the homogenous poroelastic material and displacement are plotted at the beginning and the end of the simulation (as presented on Figure 3).

In this case, the pressure reaches a homogeneous distribution. As the pressure computation considers fluid flows and fluid accumulation in the body, an increase of the pressure can be noticed in the whole volume during the simulation, when comparing the results to those obtained at the beginning and at the end of the pressure ramp. 
The increase in the pressure field agrees with the entrapment of the fluid inside the body. Moreover, it agrees with the increase of the structural stress distribution, when comparing with the results from case (ii), because of the fluid entrapment.

Indeed, when the fluid is free to flow out, the solid matrix tends to empty itself. In this case, the load is mainly supported by the structural matrix. This can be seen on Figure 5, on the pressure distribution, where it can be noticed that the internal pressure is tending to reach 0 (the reference pressure value) at the end of the simulation. At the bottom of the cube, internal stresses get higher, due to the fluid accumulation and mostly to the clamping of the bottom face, as it can also be seen on Figure 7 .

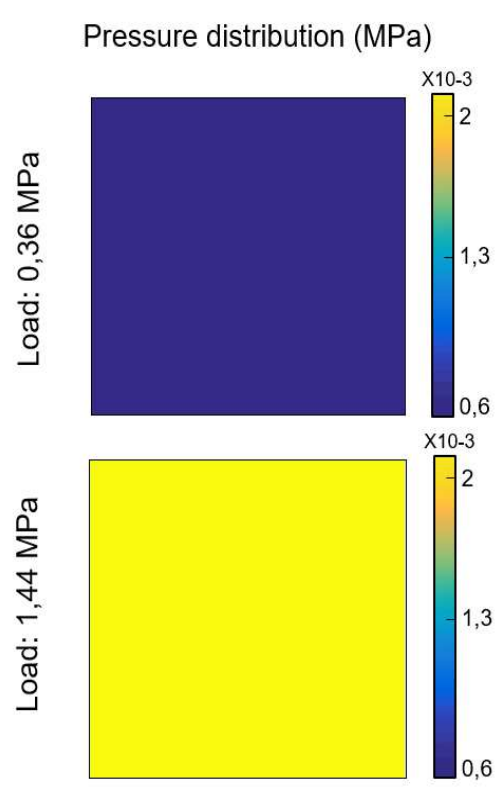

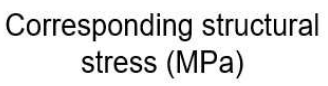

Corresponding structural stress (MPa)
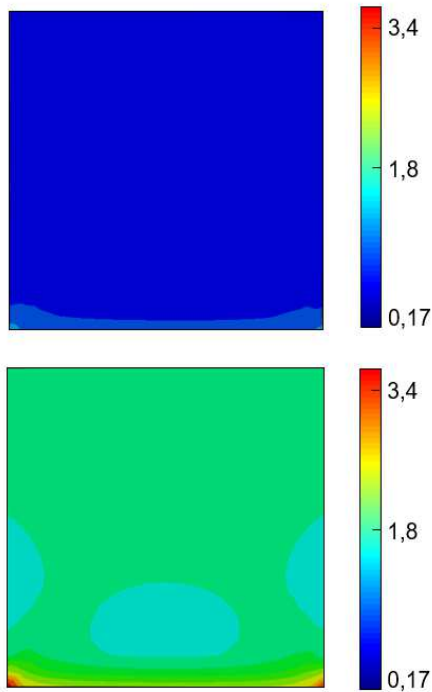

Corresponding structural displacement $(\mathrm{mm})$

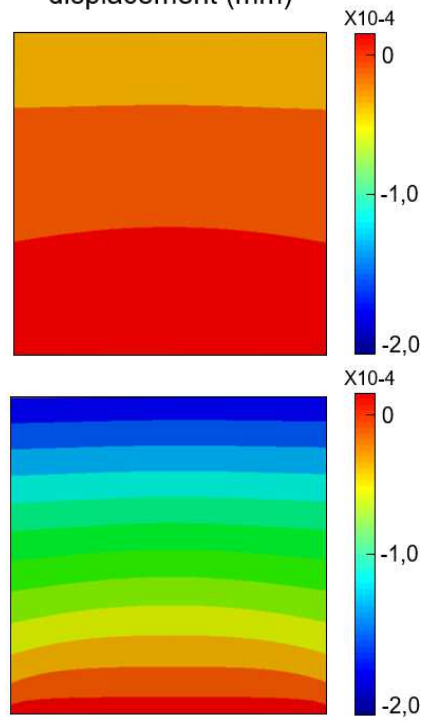

Figure 4 - Pressure distribution (MPa), the corresponding Von Mises structural stress (MPa) and structural displacement (mm) for impermeable wall boundary conditions: at 0,36 MPa (top line) and 1,44 MPa (bottom line) of applied structural load at the upper surface.

Pressure distribution (MPa)
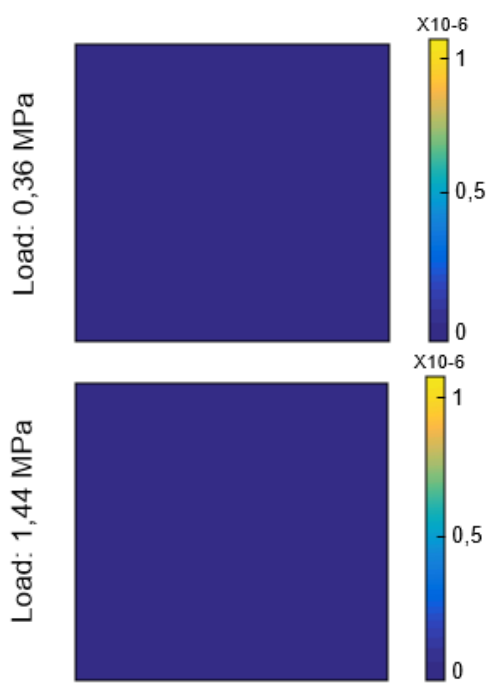

Corresponding structural stress $(\mathrm{MPa})$
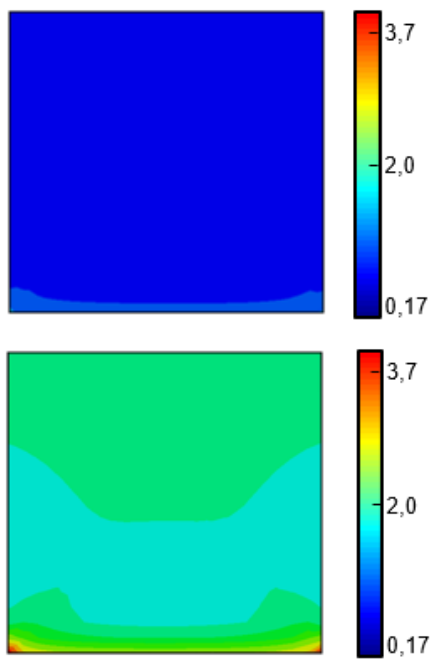

Corresponding structural displacement $(\mathrm{mm})$
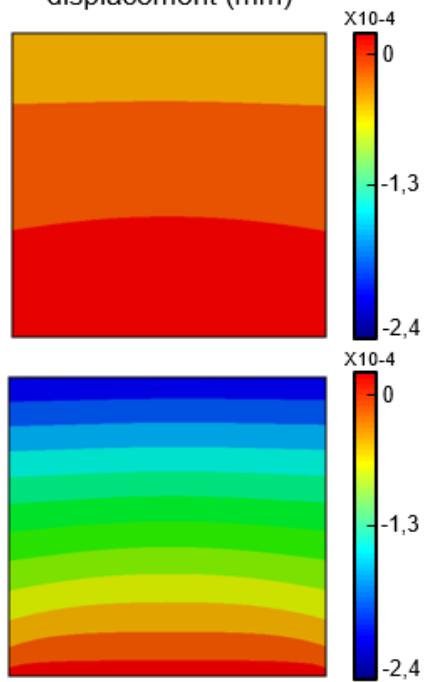

Figure 5- Pressure distribution (MPa), the corresponding Von Mises structural stress ( $\mathrm{MPa}$ ) and structural displacement (mm) for free wall boundary conditions: at 0,36 MPa (top line) and 1,44 MPa (bottom line) of applied structural load at the upper surface. 
The comparison of the pressure and stress distributions on the two cases brings to light the importance of the fluid response in the mechanical global behavior of a porous and fully saturated material, e. g. cortical bone.

In the following, to highlight the contribution of the porosity in the macroscopic mechanical response of the simulated bone, a comparison between the equivalent stiffness of an elastic and the poroelastic material has been done.

\subsubsection{Elastic stiffness vs equivalent poroelastic stiffness}

Figure 6 shows the trends of the imposed pressure as a function of the relative calculated deformation (deformation of the specimen over its total length). From the slope of such trends it is possible to extract an equivalent stiffness of the bone sample.

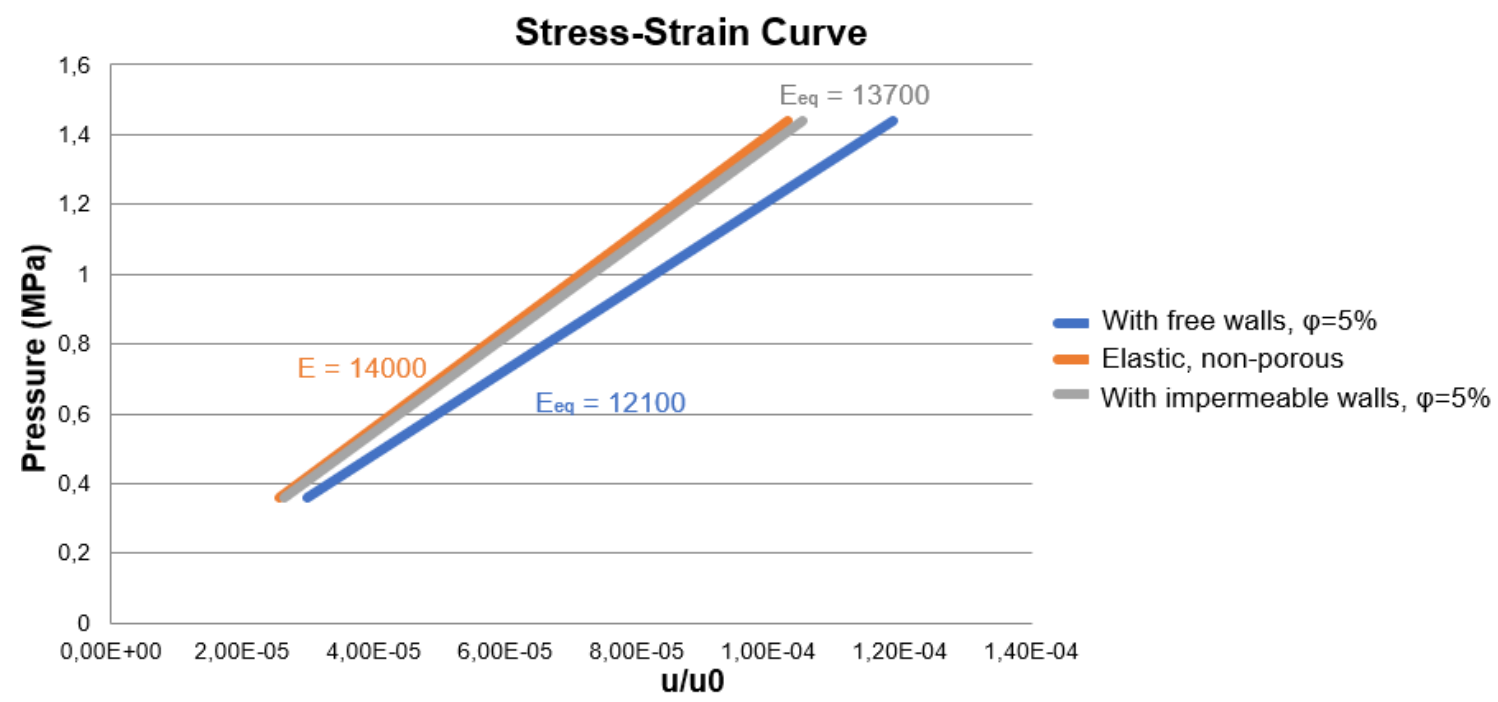

Figure 6 - Stress-Strain curves for the considered cases, with the corresponding equivalent stiffness (in MPa) calculated from the slopes of the curves.

To evaluate the mechanical behavior of the different numerical cases (boundary conditions and porosity percentage), an elastic and non-porous material, with a Young's Modulus of $14000 \mathrm{MPa}$, is reported as reference case. The structural stress distribution and the corresponding displacement are shown in Figure 7. Both distribution and values slightly higher than the ones previously presented, corroborating the influence of internal flows in the complexity of global mechanical behavior of the material. The different numerical simulations result in equivalent stiffness values, in agreement with the physical response of the poroelastic material. 


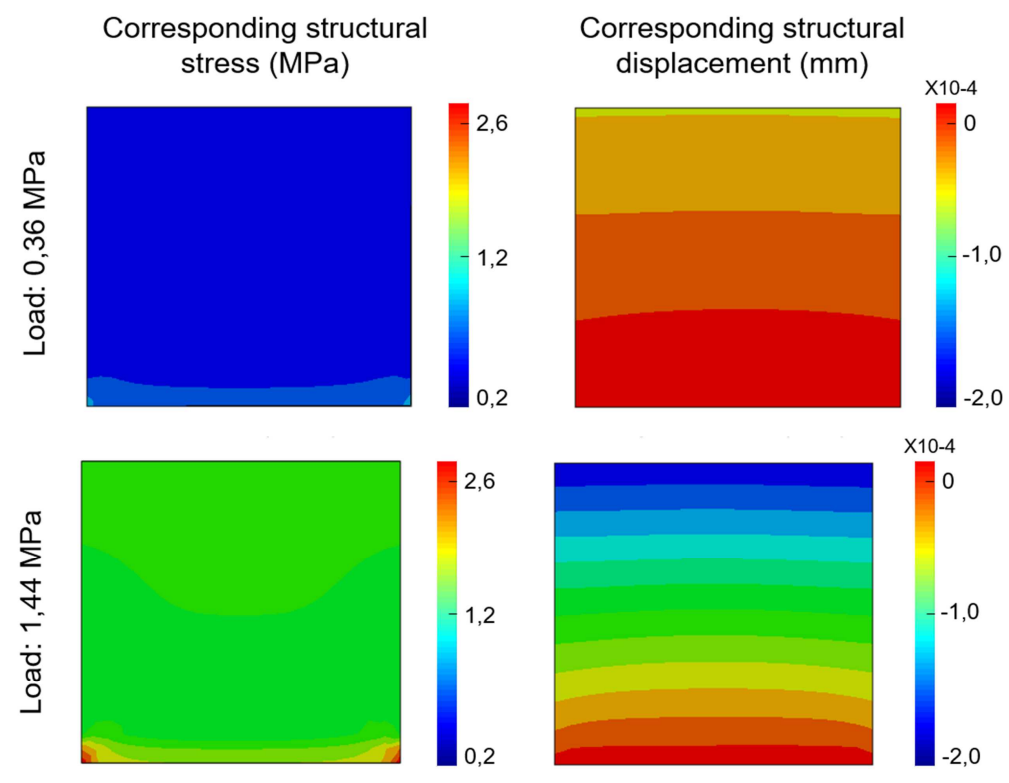

Figure 7- Structural Von Mises stress (MPa) and structural displacement in an elastic and non-porous model with a Young Modulus of 14000 MPa.

Comparing the obtained results with the case (i), when the fluid is completely trapped in the porous cube, with the elastic model, shows an equivalent stiffness almost equal to the elastic non-porous cube model, despite the changes in structural stress distribution as the internal pressure involves an increase of the structural stress at the borders of the cube.

Then, in this case, as it is plotted on Figure 6, it can be observed that the impermeable equivalent Young modulus is of $13700 \mathrm{MPa}$. In fact, because the fluid is trapped in the solid matrix, the material is almost as stiff as an elastic one, despite its porous aspect, which is in accordance with the results of case (ii) and the elastic model.

Indeed, as it is shown by the graph, the computed equivalent Young modulus is equal to $12100 \mathrm{MPa}$, when considering free lateral walls, for a reference Young modulus of $14000 \mathrm{MPa}$ (the one of the solid matrix). Thus, introducing communicating porosities and fluid flows in such material, induces a decrease of almost $14 \%$ of the equivalent stiffness.

Moreover, increasing the porosity of the material up to $40 \%$ in case (ii), the final equivalent stiffness reduces to $6500 \mathrm{MPa}$ (Figure 8 ), i.e. more than $50 \%$ compared to the non-porous model. The results agree with the expected increasing contribution of the porosity, by resulting in higher compliance of the structural matrix. 


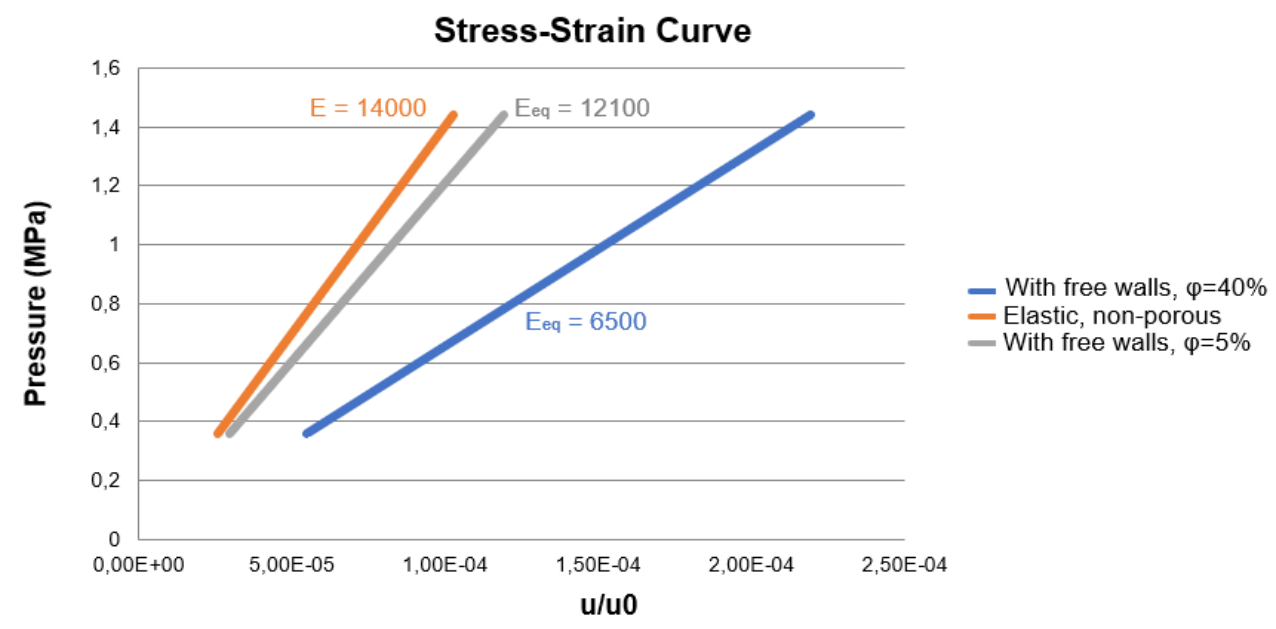

Figure 8 - Stress-Strain curves comparing case (ii) with two different porosities: 5\% (in blue) and 40\% (in orange), with the reference of the elastic case.

These opposite boundary conditions (cases (i) and (ii)), even if not truly representative of a physiological loading simulation, indicates how the poroelastic material behaves and how the internal fluid behavior affects its macroscopic stiffness. The results presented here show that considering bone as a non-porous material for ease of computation tends to overestimate its global stiffness even considering the fluid contribution, which is non-neglectable in the perspective of a structural evolution study.

Moreover, even if the chosen boundary conditions are at the extremes of a biological representation, the free wall condition is closer to experimental in vitro conditions, in which bone is tested experimentally to determine its overall mechanical properties. However, bone exact boundary conditions in the human body are extremely difficult to determine and are strongly dependent on the location, this is why the choice has been made to firstly model the two extreme sets of boundary conditions, even if it has been found in [11] that undrained condition can be a reasonable approximation. 


\section{DISCUSSION}

The model presented here lays the foundation for a mechanical approach to bone simulation based on poroelasticity, from the perspective of bone remodeling simulation accounting for the fluid contribution. The two main features on which the numerical modeling relies are multiscale behavior and fluid content within the bone. Both play a significant role on its structural and biological response.

Concerning the multiscale aspect, the homogenization technique has already proven to be numerically reliable in modeling complex materials [39] [46] [47] [48]. This technique has been used in the literature for bone modeling to account for the composite aspect of the material [49], which is particularly relevant when studying bone fracture and the influence of the mineral content of bone in the fracture process, but also accounting for its porous aspect and mechanical implications [26] [27].

As for the porous aspect, two main porosities can be distinguished in bones, in which bone fluid is involved: the vascular porosity and the lacunar-canalicular porosity. The numerical model developed here only consider the latter. Indeed, it has been suggested that the pressure in the vascular porosity is much lower than that in the lacuna-canalicular porosity and that these two porosity levels are acting independently [13] [50].

The results presented here, show that the fluid response within the lacunar-canalicular system and its intrinsic porosity have a determinant impact on bone global mechanical behavior. According to the two critical cases presented, the influence of the fluid flow at this scale plays a crucial role in bone mechanical response to compressive loading.

When considering a sample of material within the whole bone in the human body, we had a look at two extremes. The fluid will neither be free to flow out, nor will the wall be impermeable. The two simulated boundary conditions have been chosen to represent extremes of the real boundary conditions on the fluid and are not aiming to reproduce exact physiological conditions. In the perspective of an experimental compression test on a bone sample, the free wall condition at the reference pressure is to be expected.

The free wall and impermeable wall boundary conditions on pressure constitute two extreme cases that present a large scope of induced internal stresses in the poroelastic materials. The results highlight the importance of the pressure distribution in the lacunar-canalicular system, indicating that biological reality likely stands within this range of values. Indeed, the boundary pressure conditions invivo correspond to the pressure exerted by the soft tissue around the bone and the pressure within the bone structure, especially during critical loading such as climbing stairs or rising from a chair. Physically, in the thigh, the in-vivo pressure boundary conditions are more constraining than keeping the walls free, without being completely impermeable [51] [52]. Thus, the biological mechanical bone behavior must be included between these two critical cases. However, the free wall boundary condition, by contrast, will be useful for the validation through compressive tests on bone specimens.

Furthermore, as the present model aims to constitute a basis for simulation of bone remodeling, the fact that it considers the fluid flow, at the cell scale, is promising. The mechanosensitive bone cells are particularly sensitive to fluid flow [14] [2], thus this procedure would seem to lead to an interesting biologically reliable simulation of bone remodeling.

Nevertheless, some assumptions may account for the model limitations. The periodicity of the lacunarcanalicular system may seem excessive if we are getting away from a specific framework of study (cortical bone in the femur). This is the same assumption that resulted in the choice of a simple geometry in the definition of the RVE. It does not describe the very complex geometry of the lacunarcanalicular system. An interesting perspective would be an increase in the geometry complexity.

Moreover, as bone is a very complex material, both from a structural and a fluid point of view, the choice in material properties can be discussed. Indeed, bone complex hierarchical structure make difficult the choice of appropriate and accurate bone material properties at the microscale and lower according to collagen fibrils orientation and interaction with the mineral components [53]. 
Choices in fluid description can be discussed as well, as bone fluid is here considered similar to water. The presence of electrically charged components can impact bone fluid viscosity [54] and question the elimination of the Brinkman term in the fluid flow mathematical formulation. This term can be kept as the modeled fluid is considered electrically charged, to consider the electro-osmotic and osmotic contributions in nanoscopic fluid flows, and the presence of the peri cellular matrix [29] [30]. This is particularly relevant when the scope of interest is very local, which is not the case here, to keep an accurate compromise between the global model and the precise description of microscopic events. Thus, the material properties of both the fluid and the structural portions rise numerous discussions. That is why an experimentation validation of the model is required, in order to precise the values that are here or indicative purposes only.

Having developed this numerical model that accounts for bone physiological and biological reality, it can also be a useful tool in the perspective of any bone mechanical study in the future. The model is based on the critical parameters of bone porosity and the stiffness of the solid part of the bone. The last one is as well dependent on the mineralization of bone tissue, which is expected to stiffness the solid part of bone through a process that is not considered here [2]. Then, the model offers the ability to obtain information about bone intrinsic properties, through a parametric analysis for instance. It could be developed for retrieving quantitative information on the solid matrix stiffness of a tested bone sample, knowing the test conditions and updating the material properties for matching the experimental results. Obviously additional experimental interaction is required to effectively pursue this kind of study. 


\section{CONCLUSIONS}

The development of a multiscale poroelastic modelling of cortical bone has been presented. From the perspective of providing a tool to investigate bone-implant stability as a long-term goal and final aim, our choice of focusing on multiscale and poroelastic aspects of bone is relevant regarding the tendencies of the resulting model. In the case study of two borderline situations, it appears that fluid flows at the cell scale have an expected impact in bone global mechanical behavior, in reducing the equivalent stiffness of the poroelastic material according to the porosity considered.

A few conclusions may be drawn. Firstly, the fluid response plays a significant role in the structural and mechanical response of bone considering two critical sets of fluid boundary conditions. It supports the hypothesis that bone mechanical behavior cannot be determined without considering its fluid component and matches the recent finding on mechanical transduction, and the fluid role in this phenomenon.

Moreover, the developed model showed to be a possible approach able to account for poroelasticity, while being computationally efficient. The results from the two simulated borderline cases are consistent with the fluid and structural responses, providing interesting preliminary results and promising perspectives for further investigation and experimental validation

Finally, an interesting knowledge on the stiffness of the solid mineralized matrix of bone and on the equivalent stiffness can be investigated through this numerical model, to be able to distinguish the structural component from the fluid component in the bone mechanical response.

These points are critical for the elaboration of a numerical model of bone remodeling, to have an accurate representation of the mechanical transduction phenomenon and to be able to provide biologically reliable models. 


\section{REFERENCES}

[1] J.-Y. Rho, L. Kuhn-Spearing and P. Zioupos, "Mechanical properties and the hierarchical structure of bone," Medical Engineering \& Physics, vol. 20, pp. 92-102, 1998.

[2] T. M. Keaveny, E. F. Morgan and O. C. Yeh, "Bone Mechanics," in Standard Handbook of Biomedical Engineering and Design, McGraw-Hill, 2004.

[3] K. Piekarski, "Analysis of bone as a Composite Material," International Journal of Engineering Science, vol. 11, pp. 557-565, 1973.

[4] P. K. Zysset, "Indentation of bone tissue: a short review," Osteoporosis International, vol. 20, no. 6, pp. 1049-1055, 2009.

[5] P. K. Zysset, X. E. Guo, C. E. Hoffler, K. E. Moore and S. A. Goldstein, "Elastic modulus and hardness of cortical and trabecular," Journal of Blomechanics, vol. 32, no. 10, pp. 1005-1012, 1999.

[6] C. Hoffler, "An Application of Nanoindentation Technique to Measure," Journal of Biomechanical Engineering, vol. 127, no. 7, p. 1046, 2005.

[7] C. Hoffler, K. Moore, K. Kozloff, P. Zysset and M. G. S. Brown, "Heterogeneity of bone lamellarlevel elastic moduli," Bone, vol. 26, no. 6, pp. 603-609, 2000.

[8] F. Marinozzi, A. Marinozzi, F. Bini, F. Zuppante, R. Pecci and R. Bedini, "Variability of morphometric parameters of human trabecular tissue from coxo-arthritis and osteoporotic samples," Annali dell'Istituto Superiore di Sanità, no. 1, 2012.

[9] F. Marinozzi, F. Bini and A. Marinozzi, "Evidence of entropic elasticity of human bone trabeculae at low strains," Journal of Biomechanics, vol. 44, no. 5, pp. 988-991, 2011.

[10] F. Marinozzi, F. Bini, A. Quintino, M. Corcione and A. Marinozzi, "Experimental Study of Diffusion Coefficients of Water through the Collagen: Apatite Porosity in Human Trabecular Bone Tissue," BioMed Research International, vol. 2014, pp. 1-8, 2014.

[11] J. D. Gardinier, C. W. Townend, K.-P. Jen, Q. Wu, R. L. Duncan and L. Wang, "In situ permeability measurement of the mammalian lacunar-canalicular system," Bone, vol. 46, no. 4, pp. 1075$1081,2010$.

[12] R. S. Lakes, "Viscoelastic Properties of Wet Cortical Bone - I. Torsional and Biaxial Studies," Journal of Biomechanics, vol. 12, pp. 657-678, 1979.

[13] S. C. Cowin, "Bone Poroelasticity," Journal of Biomechanics, vol. 32, pp. 217-238, 1999.

[14] J. Klein-Nulend, R. Bacabac and M. Mullender, "Mechanobiology of bone tissue," Pathologie Biologie, vol. 53, pp. 576-580, 2005.

[15] N. Rosa, S. Ricardo, F. D. Magalhães and A. T. Marques, "From mechanical stimulus to bone formation: A review," Medical Engineering and Physics, vol. 37, pp. 719-728, 2015. 
[16] M. A. Biot, "General theory of three-dimensional consolidation," Journal of Applied Physics, vol. 12, pp. 155-164, 1941.

[17] M. A. Biot, "Theory of propagation of elastic waves in a fluid-saturated porous solid. II. Higher frequency range," The Journal of the acoustical Society of america, vol. 28, no. 2, pp. 179-191, 1956.

[18] J. G. Berryman, "Comparison of Upscaling Methods in Poroelasticity and Its Generalizations," Journal of Engineering Mechanics, vol. 131, no. 9, 2005.

[19] S. C. Cowin and L. Cardoso, "Mixture theory-based poroelasticity as a model of interstitial tissue growth," Mechanics of Materials, vol. 44, pp. 47-57, 2012.

[20] R. Bowen, "Theory of Mixtures," Continuum Physics, Vol. III, Mixture and EM Field Theories, pp. 1-127, 1976.

[21] A. Bensoussan, J.-L. Lions and G. Papanicolaou, Asymptotic Analysis for Periodic Structures, Elsevier, 1978.

[22] Y. Benveniste, "A New Approach to the Application of Mori-Tanaka's Theory in Composite Materials," Mechanics of Materials, vol. 6, no. 2, pp. 147-157, 1987.

[23] A. Zaoui, "Continuum Micromechanics: Survey," Journal of Engineering Mechanics, vol. 128, no. 8, pp. 808-816, 2002.

[24] L. Dormieux, A. Molinari and D. Kondo, "Micromechanical approach to the behavior of poroelastic materials," Journal of the Mechanics and Physics of Solids, vol. 50, no. 10, pp. 22032231, 2002.

[25] J. L. Auriault and D. Caillerie, "Some remarks on homogenization processes," Revue Française de Géotechnique, vol. 49, pp. 43-50, 1989.

[26] W. J. Parnell and Q. Grimal, "The influence of mesoscale porosity on cortical bone anisotropy. Investigations via asymptotic homogenization," Journal of The Royal Society Interface, vol. 6, no. 30, pp. 97-109, 2009.

[27] T. Lemaire, E. Capiez-Lernout, J. Kaiser, S. Naili and V. Sansalone, "What is the importance of multiphysical phenomena in bone remodelling signals expression? A multiscale perspective," Journal of the mechanical behavior of biomedical materials, vol. 4, pp. 909-920, 2011.

[28] S. Scheiner, P. Pivonka and C. Hellmich, "Poromicromechanics reveals that physiological bone strains induce osteocyte-stimulating lacunar pressure," Biomechanics and Modeling in Mechanobiology, vol. 15, no. 1, pp. 9-28, 2016.

[29] E. Rohan, S. Naili, R. Cimrman and T. Lemaire, "Multiscale modeling of a fluid saturated medium with double porosity: Relevance to the compact bone," Journal of the Mechanics and Physics of Solids, vol. 60, no. 5, pp. 857-881, 2012.

[30] V. Sansalone, J. Kaiser, S. Naili and T. Lemaire, "Interstitial fluid flow within bone canaliculi and electro-chemo-mechanical features of the canalicular milieu.," Biomechanics and Modeling in 
Mechanobiology, vol. 12, pp. 533-553, 2013.

[31] J. Crolet, B. Aoubiza and A. Meunier, "Compact bone: Numerical simulation of mechanical characteristics," Journal of Biomechanics, vol. 26, no. 6, pp. 677-687, 1993.

[32] C. Hellmich and F. Ulm, "Microporodynamics of Bones: Prediction of the "Frenkel-Biot" Slow Compressional Wave," Journal of Engineering Mechanics, vol. 131, no. 9, 2005.

[33] C. Hellmich, D. Celundova and F. Ulm, "Multiporoelasticity of Hierarchically Structured Materials: Micromechanical Foundations and Application to Bone," Journal of Engineering Mechanics, vol. 135, no. 5, 2009.

[34] C. Morin and C. Hellmich, "A multiscale poromicromechanical approach to wave propagation and attenuation in bone," Ultrasonics, vol. 54, no. 5, pp. 1251-1269, 2014.

[35] K. Kusuzaki, N. Kageyama, H. Shinjo, H. Takeshita, H. Murata, S. Hashiguchi, T. Ashihara and Y. Hirasawa, "Development of bone canaliculi during bone repair," Bone, vol. 27, no. 5, pp. 655$659,2000$.

[36] P. Schneider, M. Meier, R. Wepf and R. Müller, "Towards quantitative 3D imaging of the osteocyte lacuno-canalicular network," Bone, vol. 47, no. 5, pp. 848-858, 2010.

[37] O. S. T. Gbehe, M. ElKhlifi, M. Nabhani and B. Bou-Saïd, "Numerical modeling of poro-elasticity effects on squeeze film," Tribology International, vol. 102, pp. 70-78, 2016.

[38] D. Néron and D. Dureisseix, "A computational strategy for thermo-poroelastic structures with a time-space interface coupling," Internation Journal for Numerical Methods in Engineering, no. 75, pp. 1053-1084, 2008.

[39] J. L. Auriault, "Dynamic Behaviour of a Porous Medium Saturated by a Newtonian Fluid," International Journal of Engineering Science, vol. 18, pp. 775-785, 1980.

[40] H. Darcy, "Les fontaines publiques de la ville de Dijon: Détermination des lois d'écoulement de l'eau à travers le sable.," pp. 590-591, 1856.

[41] D. A. Nield and A. Bejan, "Convection in Porous Media," Springer-Verlag, 2006.

[42] H. C. Brinkman, "A calculation of the viscous force exerted by a flowing fluid on a dense swarm of particles," Journal of Applied Sciences Research, vol. 1, no. 1, pp. 27-34, 1949.

[43] W. S. Almalki and M. H. Hamdan, "Investigations in Effective Viscosity of Fluid in a Porous Medium," International Journal of Engineering Research and Applications, vol. 6, pp. 41-51, 2016.

[44] J. A. Kolodziej, "Influence of the porosity of a porous medium on the effective viscosity in Brinkman's filtration equation," Acta Mechanica, vol. 75, pp. 241-254, 1988.

[45] W. A. Hodge, R. S. Fujan, K. L. Carlson, R. G. Burgess, W. H. Harris and R. W. Mann, "Contact pressure in the human hip joint measured in vivo," Proceedings of the National Academy of Science USA, vol. 83, pp. 2879-2883, 1986. 
[46] C. Boutin and J. Auriault, "Dynamic Behaviour of Porous Media Saturated by a Viscoelastic Flui. Application to Bituminous Concretes.," International Journal of Engineering Science, vol. 28, no. 11, pp. 1157-1181, 1990.

[47] G. Allaire, "Homogenization of the Stokes flow in a connected porous medium," Asymptotic Analysis, vol. 6, pp. 203-222, 1989.

[48] U. Hornung, "Homogenization and Porous Media," vol. 6, 1997.

[49] S. Nikolov and D. Raabe, "Hierarchical Modeling of the Elastic Properties of Bone at Submicron Scales: The Role of Extrafibrillar Mineralization," Biophysical Journal, vol. 94, pp. 4220-4232, 2008.

[50] T. H. Smit, J. M. Huyghe and S. C. Cowin, "Estimation of the poroelastic parameters of cortical bone," Journal of Biomechanics, vol. 35, pp. 829-835, 2002.

[51] S. F. Bensamoun, S. I. Ringleb, L. Littrell, Q. Chen, M. Brennan, R. Ehman and K.-N. An, "Determination of Thigh Muscle Stiffness Using Magnetic Resonance Elastography," Journal of Magnetic Resonance Imaging, vol. 23, pp. 242-247, 2006.

[52] L. Debernard, L. Robert, C. F. and S. F. Bensamoun, "Analysis of thigh muscle stiffness from childhood to adulthood using magnetic resonance elastography (MRE) technique," Clinical Biomechanics, vol. 26, pp. 836-840, 2011.

[53] A. Fritsch and C. Hellmich, "Universal' microstructural patterns in cortical and trabecular,," Journal of Theoretical Biology, vol. 244, pp. 597-620, 2007.

[54] T. Abdalrahman, S. Scheiner and C. Hellmich, "Is trabecular bone permeability governed by molecular ordering-induced fluid viscosity gain? Arguments from re-evaluation of experimental data in the framework of homogenization theory," Journal of Theoretical Biology, vol. 365, pp. 433-444, 2015. 